\title{
La transmisión de los valores médicos: competencias específicas
}

\author{
Jesús Millán Núñez-Cortes ${ }^{1}$, Jorge García Seoane ${ }^{1}$, Elpidio Calvo Manuel ${ }^{1}$, Ramiro Díez Lobato ${ }^{1}$, \\ José Luis Villanueva Marcos², Angel Nogales Espert ${ }^{1}$. \\ ${ }^{1}$ Grupo de trabajo para definición de competencias de la Facultad de Medicina de la Universidad Complutense de \\ Madrid. ${ }^{2}$ Agencia Laín Entralgo para la Formación, Investigación y Estudios Sanitarios de la Comunidad de Madrid.
}

Cualquier aproximación a la definición de las competencias que el alumno debe adquirir a su paso por las Facultades de Medicina, incluye la definición y organización de aquellas que se refieren al ámbito de los valores que le son propios a la profesión médica, independizándolas y predefiniéndolas. No siempre es fácil definir y priorizar los aspectos relacionados con los valores médicos, aspectos ciertamente intangibles de la profesión, pero que resultan imprescindibles para el ejercicio de la misma. Pero la dificultad de su definición no evita la necesidad de su inclusión en el conjunto.

Así, siguiendo una estructuración clásica de las competencias a adquirir por el futuro médico, y de acuerdo con las recomendaciones nacionales e internacionales, éstas pueden referirse a aspectos relacionados con lo que "tiene que saber", "tiene que saber hacer" y "tiene que saber ser". En estos tres apartados, al menos en teoría, se podrán encontrar el conjunto de competencias relacionadas con aspectos de conocimientos, de habilidades y de actitudes.

Estos tres grupos de competencias a adquirir deben permitir al médico enfrentarse a su triple responsabilidad: frente a sí mismo (como médico), frente al enfermo (como profesional) y frente a la sociedad y el sistema sanitario (como individuo social). Al alumno, ser consciente y ser capaz de saber lo que ha de hacer en su profesión, cómo ha de hacerlo, y de que él mismo es el profesional encargado de hacerlo, le permitirá completar de forma global y unitaria su formación médica. El "qué", el "cómo" y el "quién", en el ámbito de los conocimientos, de las habilidades y de las actitudes, nos debe permitir diseñar el mapa de competencias en los estudios de medicina.
Indudablemente, de acuerdo con la categoría de la competencia y con su propia naturaleza, los distintos niveles de adquisición deben de ser formulados en términos distintos. Así, en el campo del saber, el alumno puede "reconocer, diagnosticar y orientar el manejo", ó "sólo conocer", las diferentes situaciones o problemas, dependiendo de cuál deba ser el nivel adquirido. En el campo del saber hacer, el alumno podría "hacerlo de forma autónoma" "hacerlo con tutela" ó "haberlo visto hacer" en función del nivel requerido. En el campo del saber ser, se trataría de "ser consciente", ó de "ser capaz de", según la naturaleza de la propia competencia considerada.

\section{PROYECTO FAC-MED-UCM}

La Facultad de Medicina de la Universidad Complutense ha desarrollado un polo estratégico sobre educación médica en el que una línea prioritaria ha sido la Innovación Educativa. Dicha línea estratégica ha tenido tres pilares o planes de acción preferentes: el desarrollo de la formación en habilidades, la evaluación de las competencias, a través del desarrollo de una evaluación clínica objetiva y estructurada (ECOE), y la definición de las competencias al objeto de establecer el mapa de competencias como elemento arquitectónico previo para de la definición de objetivos educativos y el diseño del futuro plan de estudios.

En una primera aproximación, las competencias han quedado agrupadas de la forma que se señala en la Tabla 1. Dentro del apartado sobre "saber quien lo tiene que hacer" se incluyen -precisamente- aquellas competencias en función de las responsabilidades personales, éticas y legales, a su vez, 


\section{Tabla 1. GRUPOS DE COMPETENCIAS}

Grupo I: Saber lo que tiene que hacer

1. Competencias en CIENCIAS BÁSICAS

2. Competencias en CIENCIAS CLÍNICAS

3. Competencias en HABILIDADES CLÍNICAS

4. Competencias en PROCEDIMIENTOS DIAGNÓSTICOS Y TERAPÉUTICOS

5. Competencias en PROMOCIÓN DE LA SALUD Y PREVENCIÓN DE LA ENFERMEDAD

6. Competencias en CIENCIAS SOCIALES

Grupo II. Saber cómo lo tiene que hacer

1. Competencias para la COMUNICACIÓN

2. Competencias para el RAZONAMIENTO, JUICIO CLÍNICO Y TOMA DE DECISIONES

3.Competencias para EL MANEJO DE LA INFORMACIÓN Y DOCUMENTACIÓN CIENTÍFICA

Grupo III: Saber quién lo tiene que hacer

1. Competencias por su RESPONSABILIDAD PERSONAL, ÉTICA Y LEGAL

2. Competencias como PROFESIONAL DEL SISTEMA SANITARIO

3. Competencias para su DESARROLLO PROFESIONAL

\section{Tabla 2. AGRUPACIÓN DE LAS COMPETENCIAS SOBRE VALORES PROFESIONALES}

\section{RESPONSABILIDAD PERSONAL, ÉTICA Y LEGAL}
A. Actitud profesional
B. Aspectos éticos
C. Aspectos legales
D. Aspectos conductuales
E. Aspectos sociales
F. Aspectos económicos
G. Aspectos profesionales

\section{PROFESIONAL DEL SISTEMA SANITARIO}
A. Sistemas de salud
B. Responsabilidades médicas
C. El médico como investigador
D. El médico como docente
E. El médico como gestor
F. El médico en un equipo

\section{DESARROLLO PROFESIONAL}
A. Autocrítica y autoevaluación
B. Aprendizaje autónomo
C. Cuidado personal
D. Carrera profesional
E. Motivación

clasificadas de acuerdo con distintos apartados, así como las relativas a las relaciones con el sistema sanitario o el propio desarrollo profesional. Tabla 2.
En función de tales apartados, se han recogido las principales competencias que podrían ser incluidas en cada uno y que se definen a continuación: 


\section{COMPETENCIAS RELACIONADAS CON LAS RESPONSABILIDADES PERSONALES, ÉTICAS Y LEGALES}

\section{A.- ACTITUD PROFESIONAL}

SER CAPAZ DE:

a) Con respecto a los PACIENTES:

- Identificarse delante del paciente

- Desarrollar una relación de confianza y respeto mutuos

- Transmitir la información teniendo en cuenta la voluntad del paciente y sus características

- Informar de manera comprensible

- Informar de la voluntariedad de las decisiones y del consentimiento por escrito

- Informar de los derechos del paciente y de sus obligaciones

- Respetar las decisiones de los pacientes sobre todo lo que le afecte

- Respetar la voluntad del paciente para información a terceros en materia que le afecte

- Poner a disposición del paciente cualquier dato de su enfermedad, si lo solicita

- Informar al paciente de la posibilidad de una segunda opinión

- Reconocer los límites del encarnizamiento terapéutico

b) Con respecto a los FAMILIARES:

- Informar del derecho del paciente a decidir el acceso a su información, y al contenido de la misma

- Informarles sobre todo lo que pueda mejorar el entorno del paciente

- Identificar las relaciones poco adecuadas entre familiares y pacientes que produzcan interferencias

c) Con respecto a los COLEGAS:

- Mantener una actitud de confianza y respeto, con predisposición a la colaboración

- Colaborar en protocolos diagnósticos y terapéuticos

\section{B.- ASPECTOS ÉTICOS}

SER CAPAZ DE:

- Tener presente la importancia y la existencia de los aspectos éticos

- Comprender lo que es y significa una relación asistencial de colaboración, no coactiva

- Comprender los deberes básicos de la medicina: el deber de respetar la autonomía

- Comprender los deberes básicos de la medicina: el deber de procurar beneficios y evitar daños

- Comprender los deberes básicos de la medicina: el deber de buscar la máxima equidad de los recursos

- Conocer y saber utilizar un método correcto en la resolución de conflictos éticos

- Comprender la importancia del derecho a la confidencialidad e intimidad, y el deber de respetarlos

- Comprender la importancia y necesidad del consentimiento informado

- Aceptar el derecho del paciente a rechazar tratamientos o actuaciones médicas, incluso vitales

- Informar sobre un paciente, respetando la debida confidencialidad

- Conocer los documentos de voluntades anticipadas y ayudar a las personas que deseen utilizarlos

- Comprender como médico que la muerte debe acontecer de acuerdo con los valores personales

- Utilizar la documentación clínica

- Facilitar un consejo genético

- Actuar correctamente cuando se conoce un error médico propio o ajeno

- Acceder y analizar los principales textos y fuentes de información de ética biomédica

\section{C.- ASPECTOS LEGALES}

SER CONSCIENTE DE:

- La normativa y documentación básica sobre requisitos para ejercer la medicina

- La normativa sobre el secreto profesional

- La normativa sobre el derecho de los enfermos

- Los delitos que se pueden cometer en el ejercicio de la medicina

- Como hace adecuadamente la valoración de las lesiones

- La intervención del médico en la valoración de incapacidades

- Las situaciones de alteración mental en su relación con capacidad, imputabilidad o peligrosidad

- La adaptación a la realidad social (costumbres, normas, leyes) de los avances de la medicina

- La identificación y genética forense con suficiencia

SER CAPAZ DE:

- Hacer el diagnóstico de muerte correctamente en las distintas circunstancias previstas en la ley

- Certificar la muerte de acuerdo con las diversas situaciones que preve la normativa

- Tener conocimientos sobre muertes no certificables, especialmente las violentas o sospechosas

- Leer textos legales e interpretarlos

- Practicar una autopsia judicial reglada

- Redactar correctamente documentos médico-legales

- Tomar muestras de un cadáver y prepararlas para su traslado

- Realizar un peritaje e informe médico-legal

- Aplicar medidas elementales de prevención de la salud laboral

- Atender a una víctima de violencia en el medio doméstico

- Realizar una investigación criminalística básica

- Realizar un peritaje de valoración de daños corporales 
- Explorar de forma básica a una presunta víctima de un delito contra la libertad sexual

- Realizar una exploración básica en un caso de sospecha de aborto criminal

- Diagnosticar (clínica y laboratorio) impregnaciones por tóxicos frecuentes en el medio laboral

- Elaborar una ficha dactilográfica y odontológica para la identificación de cadáveres

\section{D.- ASPECTOS CONDUCTUALES}

\section{SER CONSCIENTE DE:}

- El papel de la familia y amigos en la salud y en la enfermedad

- La importancia de los aspectos laborales en la salud individual

- La adecuación del individuo a las normas culturales

- La influencia del entorno físico en la enfermedad

\section{E.- ASPECTOS SOCIALES}

SER CONSCIENTE DE:

- La existencia de una sociedad multicultural

- La importancia del aprendizaje de idiomas

- Los mismos derechos y obligaciones para todos los pacientes

- La existencia de costumbres o creencias que aconsejen modificar o evitar actitudes médicas

\section{F.- ASPECTOS ECONÓMICOS}

SER CONSCIENTE DE:

- Los costos de la asistencia sanitaria

- Las limitaciones económicas de los servicios públicos de salud

- El gasto que se deriva de los actos médicos

\section{G.-ASPECTOS PROFESIONALES}

SER CONSCIENTE DE:

- El progreso que la medicina ha experimentado y experimenta

- La potencial contribución personal al progreso de la medicina

\section{COMPETENCIAS COMO PROFESIONAL DEL SISTEMA SANITARIO}

\section{A.- SISTEMAS DE SALUD}

SER CONSCIENTE DE:

- Visión y demandas de la sociedad

- Papel de los niveles de asistencia (prim.secund.terciaria)

- Los servicios sociosanitarios y paliativos de asistencia

- El papel de las distintas profesiones sanitarias

- La estructura del sistema sanitario

- El papel de la medicina privada en el sistema sanitario

- La legislación sobre el sistema sanitario

\section{B.- RESPONSABILIDADES MÉDICAS}

\section{SER CONSCIENTE DE:}

- El código deontológico

- El documento de voluntades anticipadas

- El documento de derechos y deberes de los enfermos

\section{C.- EL MÉDICO COMO INVESTIGADOR}

\section{SER CONSCIENTE DE:}

- Aplicar el método científico a la mejora de la práctica

- Importancia y necesidad de la investigación biomédica

- Importancia de la investigación como factor de progreso profesional

- Oportunidades de colaboración en la investigación en pregrado

- Necesidad de los equipos multidisciplinarios en la investigación

\section{D.- EL MÉDICO COMO DOCENTE}

\section{SER CONSCIENTE DE:}

- La necesidad de enseñar al mismo tiempo que se asiste

- La necesidad de formarse continuamente

- La responsabilidad como educador frente a la sociedad 


\section{E.- EL MÉDICO COMO GESTOR \\ SER CONSCIENTE DE: \\ - El coste económico que se deriva de su actuación profesional \\ - Mecanismos para resolver el conflicto entre recursos y necesidades \\ - Necesidad de conseguir la máxima eficacia con el mínimo coste \\ F.- EL MÉDICO EN UN EQUIPO \\ SER CONSCIENTE DE: \\ - Utilidad de aprender de otros profesionales durante su formación \\ - Utilidad de trabajar conjuntamente, desarrollando un trabajo en equipo \\ - El papel y las competencias del las profesiones sanitarias próximas}

\section{COMPETENCIAS PARA EL DESARROLLO PROFESIONAL}

\section{A.- AUTOCRÍTICA Y AUTOEVALUACIÓN}

SER CAPAZ DE:

- Comportarse de forma reflexiva y responsable, sometíendose a crítica y evaluaciones, reflexionando sobre sus resultados

- Autoevaluar sus competencias y su eficacia profesional

\section{B.- APRENDIZAJE AUTÓNOMO}

SER CAPAZ DE:

- Definir objetivos personales de aprendizaje realizables y apropiados

- Reconocer limitaciones personales e identificar áreas que precisen actualización o ampliación

- Seleccionar estrategias de aprendizaje según sus preferencias

- Usar técnicas de estudio más apropiadas y efectivas

- Usar todos los medios técnicos disponibles

\section{C.- CUIDADO PERSONAL}

\section{SER CAPAZ DE:}

- Atender a su estilo de vida, dieta, ejercicio, etc

- Requerir ayuda y consejo en situaciones difíciles

- Reconocer los peligros de automedicación y abuso de sustancias

- Conocer la influencia y prevención de factores relacionados con su trabajo para la salud propia y ajena

\section{D.- CARRERA PROFESIONAL}

SER CAPAZ DE:

- Identificar los objetivos profesionales a corto y medio plazo, los planes y las aspiraciones, trabajando para conseguirlos

\section{E.- MOTIVACIÓN}

\section{SER CAPAZ DE:}

- Reconocer los factores motivadores más relevantes y su importancia

- La definición final del grado de importancia de cada una de estas competencias, así como el nivel de adquisición de las mismas, el momento de su inclusión en el programa, la forma de adquirirlas y los métodos por el que serán evaluadas.

\section{BIBLIOGRAFÍA}

1. Basic Medical Education WFME global Standard for Quality Improvement. WFME Office. University of Copenhague. Denmark. 2003. Publicado en español en la revista Educación Médica, 7: Supl 2. pp. 7-56. 2004.

2. Declaración de Granada. (2002). Educación Médica, 5 (1), 3-5.

3. Global Minimum Essential Requirements in
Medical Education. Institute for International Medical Education Core Committee. Medical Teacher 24:130-135, 2002. Traducción al español en la revista Educación Médica, 6: 13-14 2003.

4. Libro Blanco de la Titulación de Medicina. Conferencia Nacional de Decanos de las Facultades de Medicina de España. ANECA. www.aneca.es.

5. Tuning Educational Structures in Europe. Julia González y Robert Wagenaar Eds. Universidades de Deusto y Groningen. 2003. 